\title{
Remote Two-Photon Emission From Dendritic Silver Nanoclusters
}

\author{
Jess M. Gunn, Melinda Ewald, and Marcos Dantus
}

\section{Department of Chemistry, Michigan State University, East Lansing, MI 48824}

Silver nanoparticles have been known to enhance local electromagnetic fields and therefore many optical processes, including surface-enhanced Raman scattering, second harmonic generation and multiphoton photoemission [1]. Dendritic silver nanoparticle clusters, which have a fractal nature, can behave as antennas that focus the electromagnetic field into "hot-spots" - extremely localized regions of enhanced electromagnetic fields (see Fig. 1). Recent theoretical work indicates that patterned silver nanoparticle structures could be created to enhance a field with nanometer resolution [2].

For the work presented here, silver nanoparticles were synthesized with the method presented by Lee, et al [3]. Clusters were formed using the method described by Butenko, et al., and precipitated onto quartz cover slips over a period of three days [4]. The thin film was then spin coated with a layer of poly(vinyl alcohol) to protect against oxygen and water in the air. A Ti:sapphire femtosecond laser ( $800 \mathrm{~nm}$ center, $13 \mathrm{fs}$ pulse duration, $80 \mathrm{~nm}$ bandwidth) was used in these experiments. The power was reduced to below $3 \mu \mathrm{W}$ (an experimentally observed damage threshold) before it was brought into the rear port of a Nikon TE2000-U inverted microscope and focused onto the sample with a $60 \mathrm{x} / 1.45$ NA objective. Phase distortions in the pulse, due to the objective and other optics, were compensated using MIIPS [5]. The sample was raster scanned, and the data collected with a CCD camera.

A very intriguing and surprising observation is that emission can occur at points more than $10 \mu \mathrm{m}$ from the focal spot $(\sim 0.3 \mu \mathrm{m})$. Figure 2 illustrates this phenomenon. The red shows the focal spots for which emission occurred locally - within $1 \mu \mathrm{m}$. The green shows the focal spots for which emission occurred at $6 \mu \mathrm{m}$. The blue shows the focal spots for which emission occurred remotely $-10 \mu \mathrm{m}$. Understanding the nature of the energy transfer that is clearly taking place within the silver nanoparticle structure will allow insight into both the architecture of such dendritic silver and in its potential use in creating a controlled mechanism by which energy can be transferred from one point to another.

[1] S. Link and M. A. El-Sayed, Annu. Rev. Phys. Chem. 54 (2003) 331.

[2] M. I. Stockman, S. V. Faleev and D. J. Bergman, Phys. Rev. Lett. 88 (2002)

[3] P.C. Lee and D. Meisel, J. Phys. Chem. 86 (1982) 3391.

[4] A. V. Butenko, V. M. Shalaev, M. I. Stockman, Z. Phys. D. (1988) 81.

[5] B. Xu, J. M. Gunn, J. M. Dela Cruz, V. V. Lozovoy and M. Dantus, J. Opt. Soc. Am. B. (2006) (in press). 


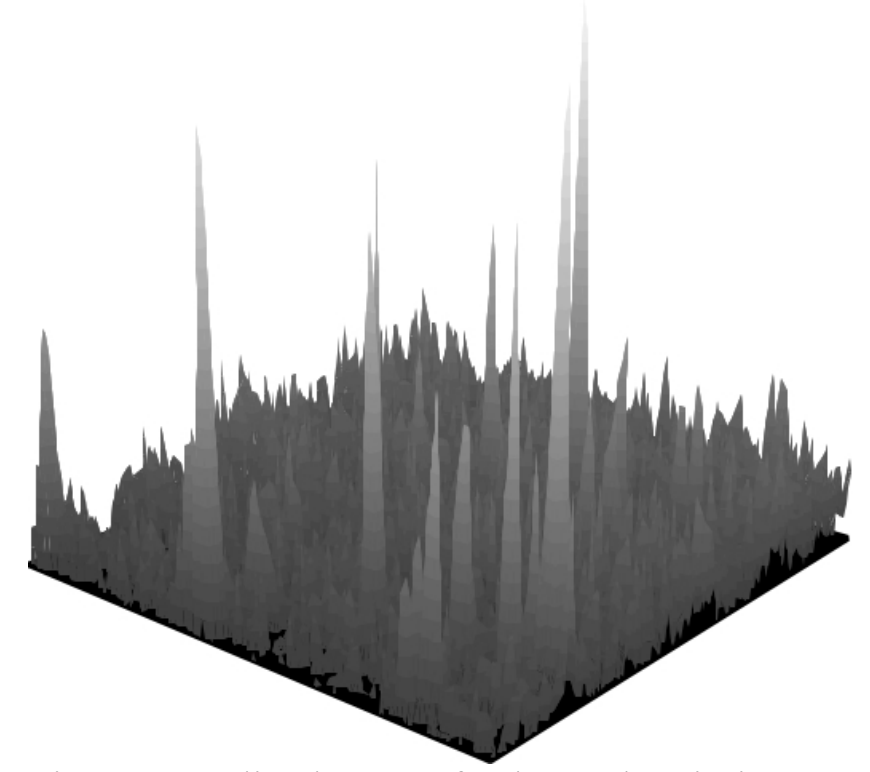

Fig. 1. Localized areas of enhanced emission on a thin film of silver dendrimer. One side is 30 microns in length.

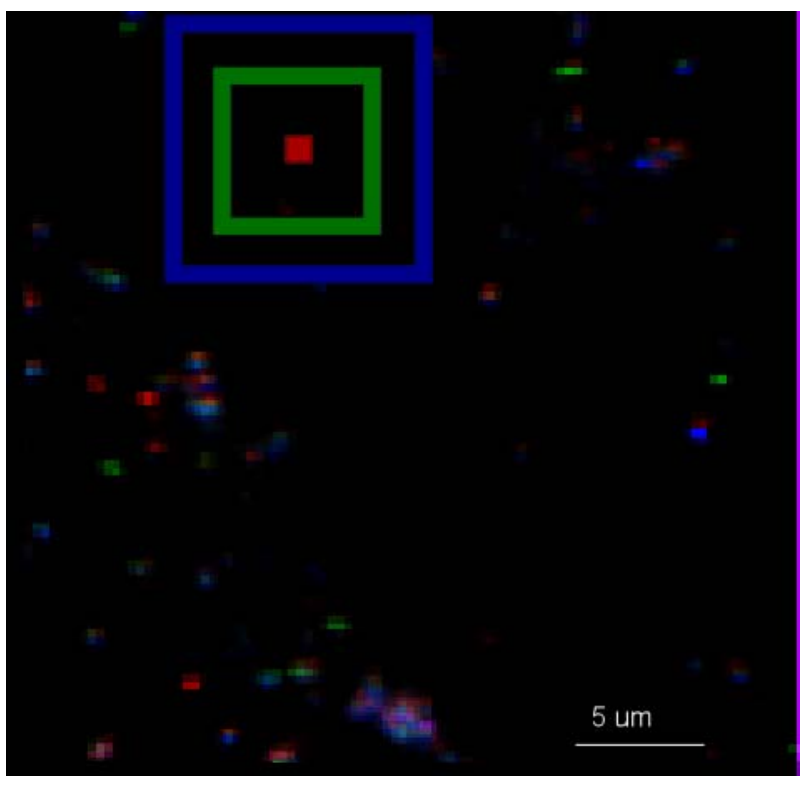

Fig. 2. Local versus remote emission. The red spots indicate focal spots that prompted emission within $1 \mu \mathrm{m}$. The green spots indicate focal spots that prompted emission at $6 \mu \mathrm{m}$ away. The blue spots indicate focal spots that prompted emission at $10 \mu \mathrm{m}$ away. 